\title{
Near-IR Integral Field Spectroscopy of the central region of NGC 5929
}

\author{
Rogemar A. Riffel ${ }^{1}$, Thaisa Storchi-Bergmann ${ }^{2}$ and Rogério Riffel ${ }^{2}$ \\ ${ }^{1}$ Universidade Federal de Santa Maria, Departamento de Física/CCNE, 97105-900, Santa \\ Maria, RS, Brazil. \\ email: rogemar@ufsm.br \\ ${ }^{2}$ Universidade Federal do Rio Grande do Sul, Instituto de Física, CP 15051, Porto Alegre \\ 91501-970, RS, Brazil. \\ email: thaisa@ufrgs.br; riffel@ufrgs.br
}

\begin{abstract}
We present two-dimensional (2D) near-infrared spectra of the inner $300 \times 300 \mathrm{pc}^{2}$ of the Seyfert 2 galaxy NGC 5929 at a spatial resolution of $\sim 20$ pc obtained with the Gemini Near infrared Integral Field Spectrograph (NIFS). We present 2D maps for the emission line flux distributions and kinematics and report the discovery of a linear structure $\sim 300 \mathrm{pc}$ in extent and of $\sim 50 \mathrm{pc}$ in width oriented perpendicular to the radio jet, showing broadened emission-line profiles.

While over most of the field the emission-line profiles have full-widths-at-half-maximum (FWHM) of $\sim 210 \mathrm{~km} / \mathrm{s}$, at a linear structure perpendicular do the radio jet the emissionline FWHMs are twice this value, and are due to two velocity components, one blueshifted and the other redshifted relative to the systemic velocity. We attribute these velocities to an outflow from the nucleus which is launched perpendicular to the radio jet. We reported the detection of this peculiar outflow in Riffel, Storchi-Bergmann \& Riffel (2014a), where more details of the analysis can be found. Since, NGC 5929 has a Type 2 nucleus, this detection implies that: (1) both ionizing radiation and relativistic particles are escaping through holes in the torus perpendicular to the radio jet; and/or (2) the torus is also outflowing, as proposed by recent models of tori as winds from the outer parts of an accretion flow; or (3) the torus is absent in NGC 5929.

At other locations the gas kinematics is dominated by rotation in a disk, although some evidences of interaction of the radio jet with the emitting gas are seen as a broadening of the line profiles at the locations of the radio structures.

The flux distributions for the $[\mathrm{P}$ II $],[\mathrm{Fe}$ II $], \mathrm{H}_{\mathrm{I}}$ and $\mathrm{H}_{2}$ emission lines show that the line emission is more extended along the $P A=60 / 240^{\circ}$, extending to up to $1.5^{\prime \prime}$ to both sides of the nucleus, while to the perpendicular direction $\left(P A=-30 / 150^{\circ}\right)$ the emission is extended to $0.7^{\prime \prime}$ from the nucleus. The flux distributions of all emission lines show a good correlation with radio the radio structures, with the two peak of emission associated to the soutwestern and northeastern radio knots. Some differences are observed among distinct emission lines. While the $[\mathrm{Fe} \mathrm{II}]$ and $\mathrm{H}_{2}$ emission peak at the location of the soutwestern radio structure at $0.6^{\prime \prime}$ from the nucleus, the $\mathrm{H}$ I recombination lines present the their highest fluxes at the location of the northeastern radio hotspot at $0.5^{\prime \prime}$ from the nucleus. Another difference is that the $\mathrm{H}_{2}$ emission is less collimated than that for other lines, being more extended perpendicularly to the radio jet. A detailed analysis of the line emission and kinematics will be presented in Riffel, Storchi-Bergmann \& Riffel (2014b).
\end{abstract}

\section{References}

Riffel, R. A., Storchi-Bergmann, T., \& Riffel, R. 2014a, ApJ, 780, 24.

Riffel, R. A., Storchi-Bergmann, T., \& Riffel, R. 2014b, in preparation. 